\title{
El Día de la Luz II (The Day of Light II): optics demonstration for high school students
}

Chaitanya S., Manoj Mathew, Giorgio Volpe, Osamu Takayama, Alejandra Valencia

Chaitanya Kumar S., Manoj Mathew, Giorgio Volpe, Osamu Takayama, Alejandra Valencia, "EI Día de la Luz II (The Day of Light II): optics demonstration for high school students," Proc. SPIE 9666, 11th Education and Training in Optics and Photonics Conference, 966603 (5 June 2009); doi: $10.1117 / 12.2207772$

Event: Eleventh International Topical Meeting on Education and Training in Optics and Photonics, 2009, St. Asaph, United Kingdom 


\title{
El Día de la Luz - II (The Day of Light -II) - Optics Demonstration for High School Students
}

\author{
S.Chaitanya Kumar, Manoj Mathew, Giorgio Volpe, Osamu Takayama and Alejandra Valencia. \\ ICFO-The Institute of Photonic Sciences, Mediterranean Technology Park, \\ Av. del Canal Olmpic s/n 08860 Castelldefels (Barcelona), Spain \\ email: chaitanya.suddapalli@icfo.es
}

\begin{abstract}
Photonics is an upcoming field that offers immense possibilities in frontier science, technology, and industry. The topic needs to be introduced among the young students to motivate their interest and passion for light. However, the potential of optics and photonics as a very exciting part of science is not always fully explored in high school education. With the motivation to contribute an initiative along these lines, a two-hour program was developed and successfully implemented at ICFO-The institute of photonics sciences. Further recent efforts were directed towards the improvement of this program which resulted in the advanced version. This improved version focuses on explaining the ray and wave nature of light, as well as the demonstration of the conservation of energy in relation to optics. The event was organized and the demonstrations were carried out by ICFO PhD students enrolled in the ICFO Optical Society of America (OSA) and SPIE student chapters.
\end{abstract}

Keywords: Optics education, Optics experimental demonstrations, Educational outreach, Fascination of light.

\section{INTRODUCTION}

Science and technology of photonics have revolutionized almost every aspect of our life in the last decade. From the use of lasers in CD players and bar code readers to very high speed data communication through optical fibers, photonics has provided many a advances in fields ranging from entertainment to information technology. On the other hand using tools of photonics, scientists have gained insight into many aspects of fundamental science ranging from quantum physics to biology and medicine. In the coming years, the field of photonics will be full of advancements with newer optical tools, techniques and devices that are being introduced at a very rapid rate ${ }^{1}$.

One prime requirement to sustain such an explosive growth in photonics is well trained workforce. Hence training in photonics is of utmost importance. At the graduate level many efforts are devoted to this aim. Many universities have been building up infrastructure to introduce new courses in photonics and optical engineering and upgrading the curriculum in traditional physics and engineering courses to introduce topics in photonics.

This effort in building up workforce in photonics needs a sustained effort starting from an early stage, specifically at the high school level. High school is the time when students make career choices. If universities need to attract talented students to these efforts, at the high school level much has to be done in creating awareness and interest for this field. Photonics needs to be introduced among the young students to motivate their interest and passion for light. However, the potential of optics and photonics as a very exciting part of science is not always fully exposed in high school education. There are two main reasons for this: 1) Optics that forms the very basics of photonics, in general, is not a part of high school science curriculum and 2) schools lack resources to introduce optics through experimental demonstrations that would introduce optics as an exciting applied science. In schools which do teach optics, very often the teaching material is not sufficiently comprehensive. The lack of awareness among high school students that optics is now in the forefront of technology and that it can open up many avenues for future careers, has driven some talented students away from taking up photonics related courses at the graduate level. However, simple experimental demonstrations, invoking various skills like, observing, comparing, describing, inferring and predicting are excellent tools to make optics a topic of interest among the youth ${ }^{2,3}$. 
With the motivation to contribute an initiative in creating awareness about optics, its prospects as the foundation of photonics and the immense possibilities offered by this field, a two-hour program for secondary school students, "El día de la luz”, was developed and implemented earlier at ICFO and various schools in Barcelona ${ }^{4}$. On successful implementation of the program, we further focused our efforts towards the introduction of some key concepts, resulting in an advanced version of the program, "El día de la luz-II". This version of the program explains the ray and wave nature of light, as well as the demonstration of the conservation of energy in relation with optics.

\subsection{ICONS}

ICONS stands for "ICFO Network and Organization of Students," based at ICFO-The Institute of photonic sciences, Barcelona, Spain ${ }^{5}$, and is the joint student organization of OSA and SPIE student chapters. A number of post-graduate students from more than 15 countries, with light as their passion, are enrolled in OSA and SPIE chapters as a part of ICONS ${ }^{6}$. ICONS organizes a variety of events like weekly colloquium by researchers from ICFO, coffee sessions with eminent scientists in the field of optics and photonics, regular laboratory visits for students from various universities, science week in Barcelona, etc. As a part of the local outreach activities, it has been actively contributing to the promotion of optics and photonics among young students through programs like "El día de la luz" (The day of light). ICFO-OSA student chapter, which is now a part of ICONS, is also the founder "International Network of OSA Student chapters", (IONS) ${ }^{7}$, an exchange program among OSA student chapters to meet optics students all over the world by networking and opening up new avenues for international collaborations.

ICONS has also played a significant role in setting the stage for the "Fascination of Light". Fascination of light is an itinerary exhibition that aims at bringing the physics and technologies based on light to the general public and school kids. The age range for the participants is from 8 years old until 17 years and also for public not directly involved in science ${ }^{8,9}$.

Fascination of light is an initiative of the German ministry of education and the European Union. The exhibit has travelled around various cities in Europe and at the end of year 2008, the exhibition visited Barcelona. It was hosted at ICFO and a great part of the logistics of this event was the responsibility of ICONS. In Barcelona, fascination of light had more than 1200 visitors in a three weeks period. The visits were organized in such a way that the schools set an appointment to visit the exhibit. At ICFO we had two visits per day. The exhibit was distributed in 5 main sections and small groups of around eight
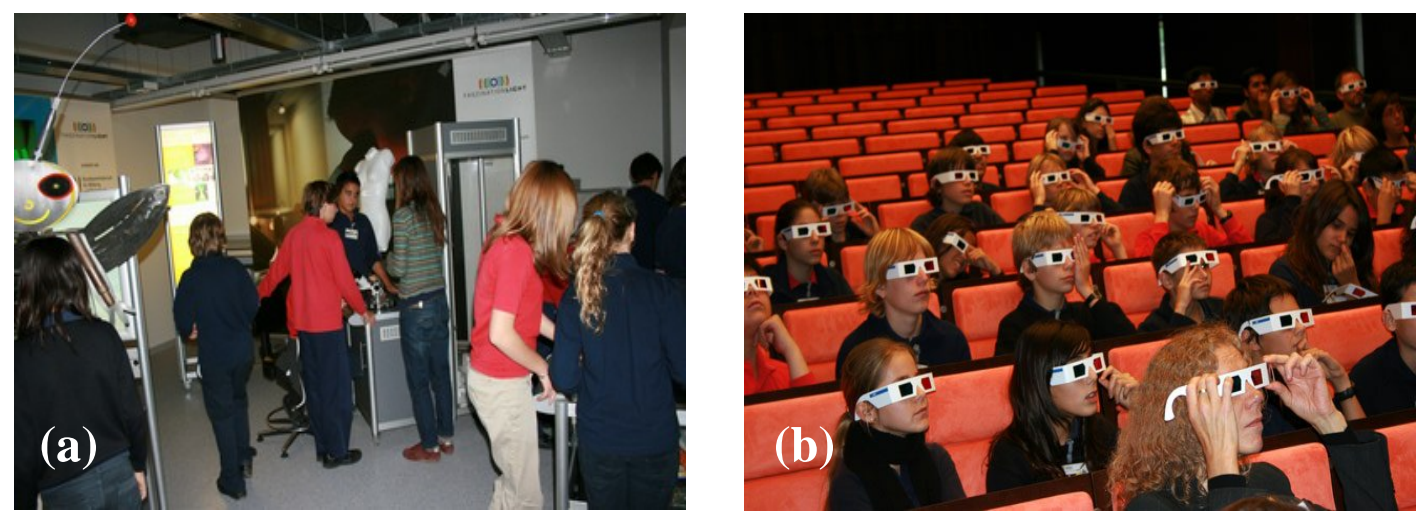

Fig. 1. Students (a) participating in fascination of light and (b) experiencing the 3D vision.

people were visiting each room at a time. In each room an ICONS member was in charge of explaining the different displays of the exhibition. The visit lasted about 90 minutes. As an original part of the hosting of fascination of light in Barcelona, it is worth mentioning that before bringing the visitor to the exhibit a small introduction to ICFO and to some of the fascinating 
issues behind light was presented. In this introduction a brief explanation of why we are able to see in three dimensions (3D) was given based on games with the eyes, as shown in Fig. 1(a, b). Finally, some 3D pictures were shown and the appropriate glasses to see them were given. The content of this introduction was taken from activities that are done by the student chapter in the "El día de la luz" program.

\section{EL DÍA DE LA LUZ (THE DAY OF LIGHT)}

With motivation to expose secondary school students to the field of optics and photonics using daily applications, we developed and implemented a two hour-long hands-on introduction to optics for high school students under the name "El día de la luz". In this workshop, we started by instigating some fundamental questions such as, origin of light, various sources of light and its applications in our daily life, after which we introduce the basic concepts of light, namely, wavelength, reflection, refraction, polarization, and stereoscopic vision. The concepts of reflection and refraction are then used to practically demonstrate total internal reflection and its application in guiding light through an optical fiber. The introduction to polarization is used to explain the working principle of liquid crystals and their application in various optical and electronic gadgets. The stereoscopic vision is practically demonstrated by 3D images and 3D glasses, making the students realize the need for two eyes ${ }^{4}$. This program has the advantage of not being very lengthy, which allows school teachers and students to attend it without sacrificing regular curricular activities. The workshop does not require sophisticated equipment so that teachers and students can easily learn how to reproduce the demonstration themselves with the help of take home materials, such as liquid crystal cells and 3D pictures, provided during the event.

\section{EL DÍA DE LA LUZ - II (THE DAY FO LIGHT - II)}

This section deals with the improved version of "El día de la luz” for high school students. The event starts with a general introduction to optics and describing the evolutionary aspects of light starting from the particle nature of light as proposed by Empedocles, Pythagoras and Isac Newton. Further more, light as wave is presented in conjunction with the classic works of

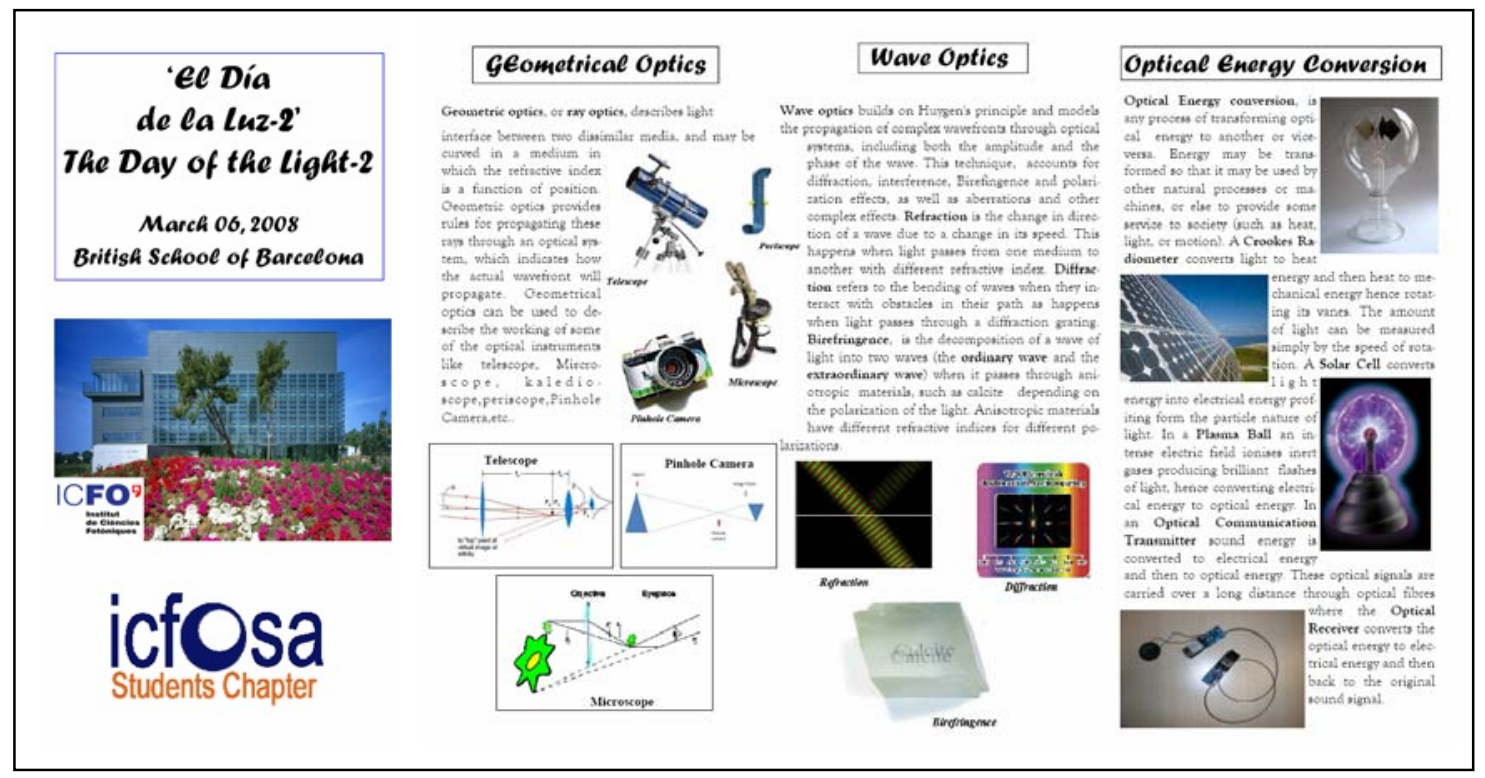

Fig. 2. A brochure of El día de la luz-II provided to the students.

Christian Huygens, James Clerk Maxwell and Heinrich Hertz. The dual nature of light is then described by referring to the discoveries by Max Planck and Albert Einstein. This method of approach not only gives the students a perspective for the 
evolution of light in the way it is understood today, but also makes them aware of renowned mathematicians and physicists who made significant contributions for the advancement of the science of light. Based on the discussions in the introduction, the demonstrations are classified into three categories as shown in Fig. 2.

They are,

1. Geometrical optics

2. Wave optics

3. Optical energy conversion

Then the students are divided into three groups to undergo the demonstrative sessions, giving insight using simple experiments. They are also provided with a leaflet that summarizes our demonstrations as shown in Fig. 2. Some of the demonstrations used in this program are home made and some are from the "Optics Suitcase" 10 .

\subsection{Geometrical optics}

First of all, basic concepts of geometrical optics are introduced using a few slides. The students learn that geometrical optics, or ray optics, describes light propagation in terms of "rays": rays are bent at the interface between two dissimilar media, and may be curved in a medium in which the refractive index is a function of position. Geometrical optics provides rules for propagating these rays through an optical system, which indicates how the actual wave-front will propagate and are very useful in everyday life at the moment of designing a pair of glasses, for instance.

Geometrical optics, moreover, can be used to describe the working principle of some of the optical instruments like telescope, microscope, kaleidoscope, periscope and pinhole camera. All these devices are usually well known to the students, but they do not usually know how they work. Practical demonstration of their working principle helps them visualizing the concepts that have been introduced previously on the slides. A gadget pen, made of two lenses, allow us to explain at the same time a very basic example of a telescope and a microscope and how light rays that form an image can be expanded or compressed by using a couple of lenses with different focal lengths, looking from one side to the other small objects are magnified, while farther objects can be magnified when reverting the order of the lenses in front of the eye.

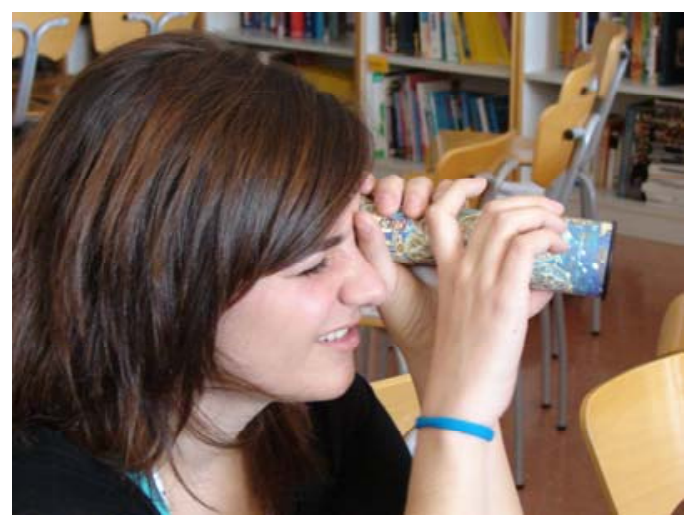

Fig. 3. A student playing with the kaleidoscope

A student playing with the kaleidoscope is shown in Fig. 3. The periscope is again a good tool to explain the ray approximation in Geometric Optics: the students can see how mirrors can bend and direct light, guiding the formation of images. A pinhole camera is a very simple camera with no lens and a single very small aperture. Simply explained, it is a light-proof box with a small hole in one side. Light from a scene passes through this single point and projects an inverted 
image on the opposite side of the box. Cameras using small apertures, and the human eye in bright light, both act like a pinhole camera. Again this can easily be explained to the students interpreting it according to the ray description of light used in Geometrical Optics. In our case the pinhole camera is a home made one. We use a carton box with a small hole which projects the inverted image on a white paper.

\subsection{Wave optics}

In the part of wave optics, we conduct a series of hands-on experiments to demonstrate the wave nature of light such as polarization, birefringence, and interference of light with diffraction gratings.

We start with a review of previous "El Día de la Luz”, that is, refraction, reflection, and polarization. To demonstrate refraction, we dip a pen in water in which the pen looks bent in the water due to refraction as shown in Fig. 4(a). By this simple example, students realize that light changes its direction when light passes from one medium to another with different refractive index.

We used a hemi-cylindrical plastic container with a protractor on top and a laser beam to demonstrate the total-internal reflection. Following the procedure shown in Fig. 4(b), the students are able to measure the critical angle for different liquids such as water and juice.
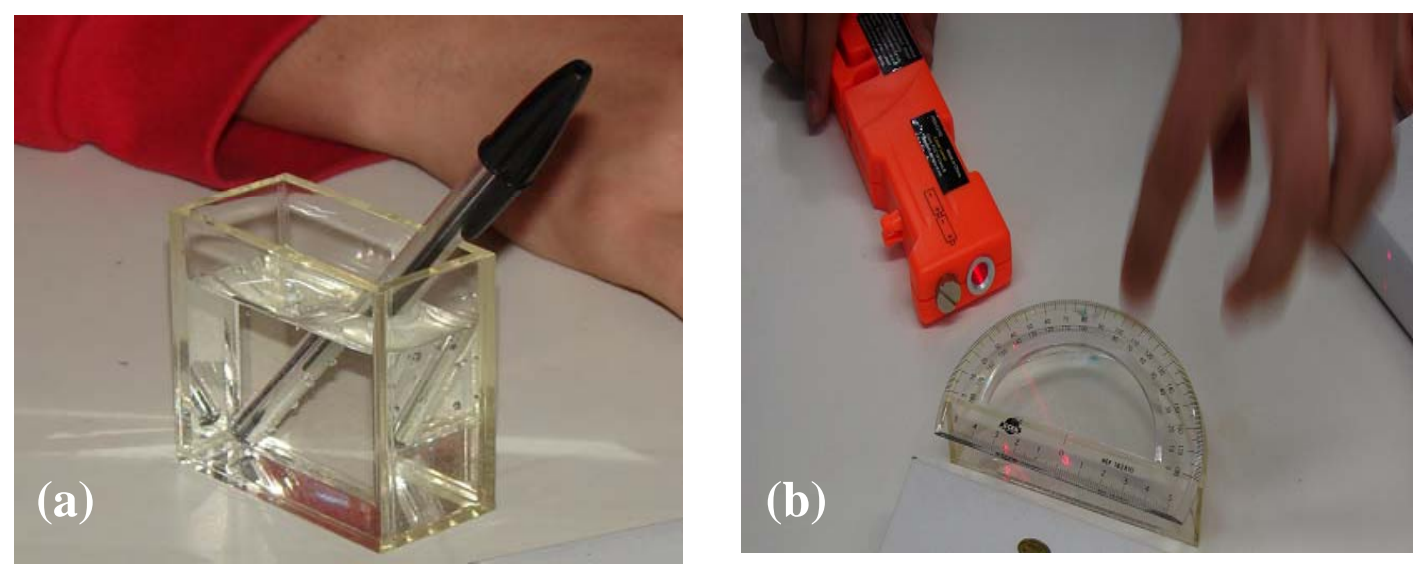

Fig. 4. Simple experiments to demonstrate (a) refraction of light and (b) total internal reflection.

With plastic polarizers, students learn that light as wave has two orthogonal direction of oscillation called polarization and observe that light cannot pass through two crossed polarizers.

We teach them with slides that material can be birefringent or anisotropic and light with different polarization can propagate differently. This is because birefringence causes decomposition of a wave of light into two waves (the ordinary wave and extraordinary wave) when it passes through anisotropic materials such as calcite, depending on the polarization of the light. Anisotropic materials have different refractive indices for different polarizations.

Later on they were introduced to the new demonstration of birefringence with calcite crystal. When you place a calcite crystal on the newspaper, double letters are seen as shown in Fig. 5(a). Then, students are asked to put a polarizer and realize that one of the images disappears as image with one of the polarizations is blocked by the polarizer. As students laterally rotate the polarizer the current images disappears and other image appears. Another characteristic of the wave nature of light is interference. To introduce this concept we use slides and a movie. We then explain that waves from two point sources can interact and create interference patterns ${ }^{11}$. With plastic diffraction gratings as in Fig. 5(b), we demonstrate that laser beam 
splits into several spots after the grating due to the diffraction. Moreover, students observe that light with different colours diffract by different amounts, red diffracts more than green.
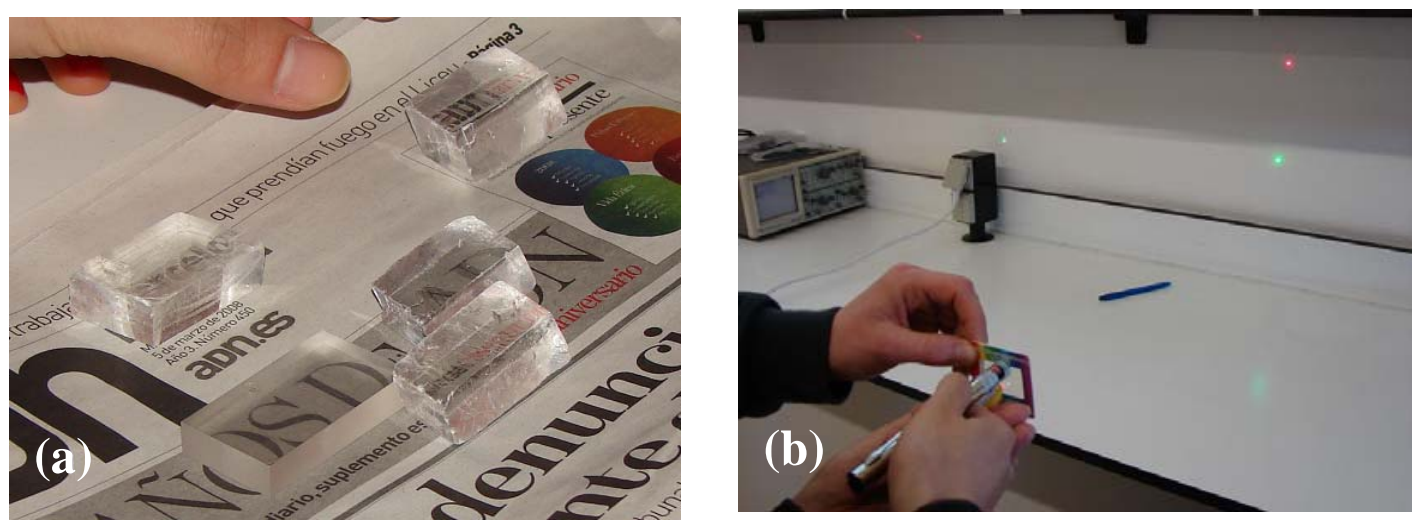

Fig. 5. Experiments to demonstrate (a) birefringence using calcite crystal and (b) diffraction from grating.

We pass diffraction gratings and flash lights that emit white light to students and made of the plastic diffraction gratings from 'Optics Suitcase' provided by OSA to students ${ }^{10}$. They shine white light through the plastic diffraction grating, and see that white light is composed of different colours. Finally, students wear glasses made of diffraction gratings and enjoy seeing various colours of light from white light sources.

\subsection{Optical energy conversion}

This session constitutes a set of four demonstrations, as listed below, explaining the conversion of energy from one form to another in various applications of light as shown in Table. 1.

\begin{tabular}{|c|c|c|c|}
\hline Sl.No. & \multicolumn{2}{|c|}{ Demonstration } & Energy conversion \\
\hline 1 & \multicolumn{2}{|c|}{ Radiometer } & Light energy $\rightarrow$ Heat energy $\rightarrow$ Mechanical energy \\
\hline 2 & \multicolumn{2}{|c|}{ Solar cell } & Light energy $\rightarrow$ Electrical energy $\rightarrow$ Mechanical \\
\hline 3 & \multicolumn{2}{|c|}{ Plasma ball } & \multicolumn{2}{|c|}{ Electrical energy $\rightarrow$ Light energy } \\
\hline 4 & $\begin{array}{c}\text { Optical } \\
\text { communication }\end{array}$ & Transmitter & Sound energy $\rightarrow$ Electrical energy $\rightarrow$ Light energy \\
\cline { 4 - 4 } & & Receiver & Light energy $\rightarrow$ Electrical energy $\rightarrow$ Sound energy \\
\hline
\end{tabular}

Table. 1. Energy conversion in to various forms in different experimental demonstrations.

The session starts with the demonstration of Crook's radiometer as shown in Fig. 6(a), where a student is asked to shine a flash lamp on the wings of a radiometer and observe that the wings start rotating in the presence of light. This makes them understand that some energy is imparted to the wings in the form of heat by the light from the flash lamp, which in turn makes them rotate. Thus, showing the conversion of light energy to heat energy and then to mechanical energy. The students then proceed to experiment with solar cell as in Fig. 6(b), where the solar cell, on illumination using a flash lamp switches ON a motor connected to it. The energy conversion here is explained with the help of Einstein's photoelectric effect. 

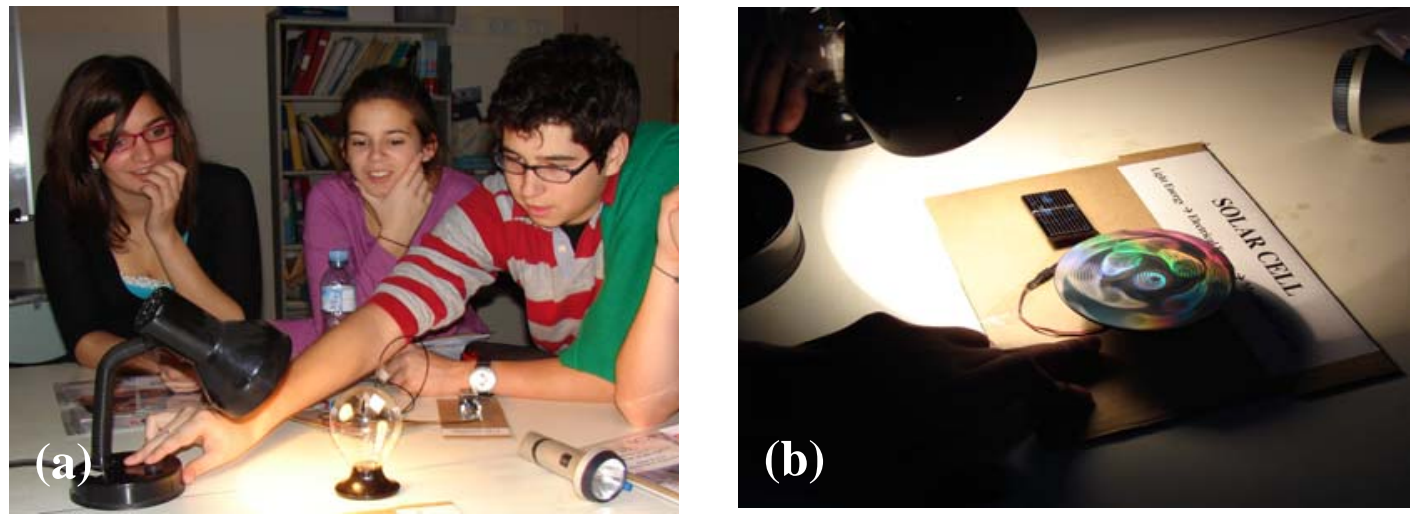

Fig. 6. Demonstration of optical energy conversion using (a) crook’s radiometer and (b) solar cell.

The Plasma ball shown in Fig. 7(a) is the most attractive part of the session. Although it was difficult for them to understand about the creation of plasma and the discharge occurring, it developed the curiosity in the students along with fun. Finally the students are introduced to the modern fiber optic communication system with the help of a home made prototype shown in Fig. 7(b), which consists of a transmitter, a fiber optic channel and a receiver. The individual parts of the system are
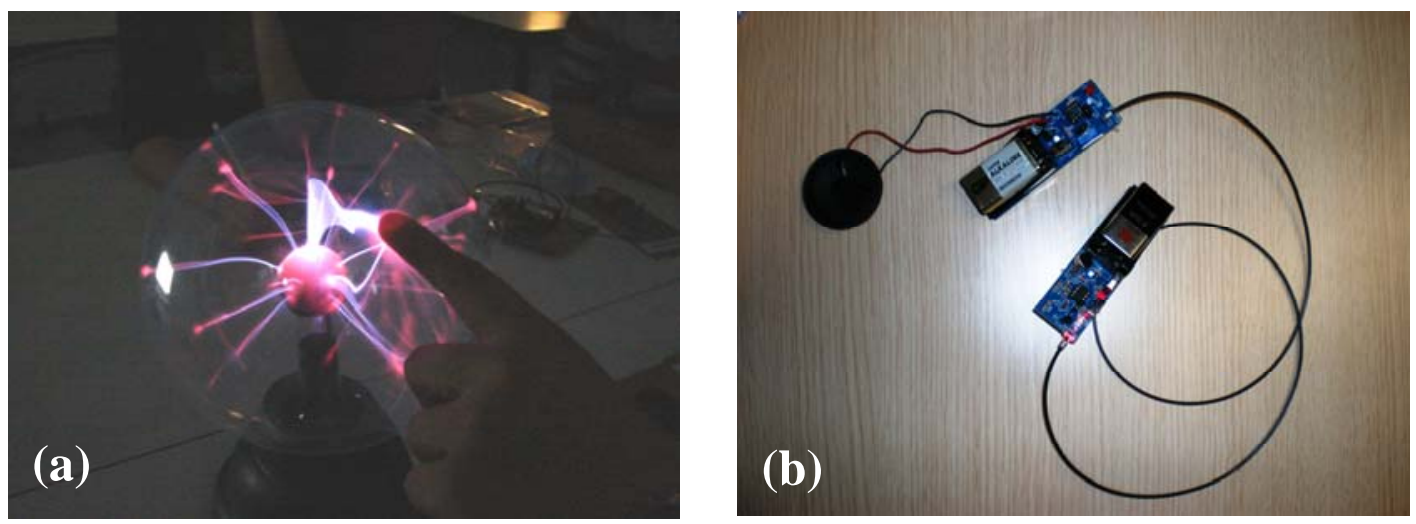

Fig. 7. Energy conversion explained using (a) Plasma ball and (b) home-made optical communication kit.

explained and students are asked to connect the fiber channel between transmitter and receiver so that they can communicate among themselves with it. This demonstration not only explains the conversion of energy and guiding light, but also gives them an insight in to the technologies they use in their daily life.

\subsection{Concluding remarks}

The program is concluded with a review of all the experiments demonstrated, by posing some simple questions. The students actively participated in this session by inferring interesting concepts from their observations. The organizing team of the program, shown in Fig. 8, helped the students in answering the questions. After this questions-answer session, the students are shown a video, "Careers in Optics"12. This video allows the students to experience the excitement of people working in the fascinating and rapidly expanding fields of optics and photonics, thus projecting optics as an exciting profession with a bright future and contains explanation of optics and photonics technologies, examples of its many applications, interviews 


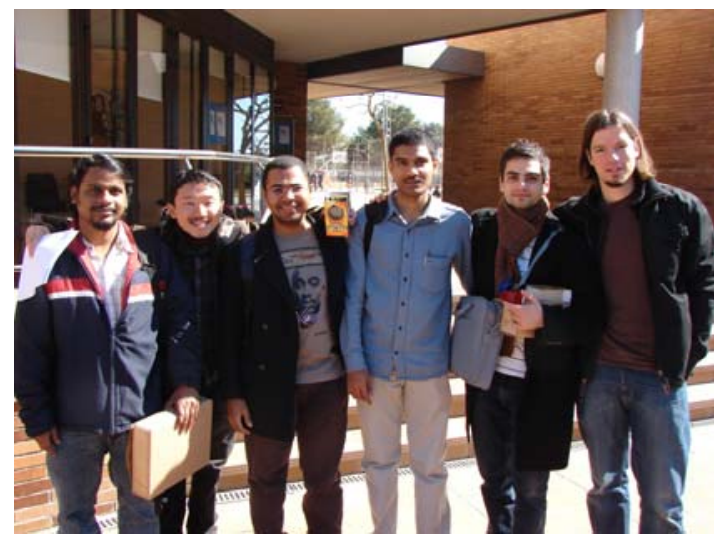

Fig. 8. Team of instructors participated in "El día de la luz-II” at a local school in Barcelona.

with enthusiastic and diverse individuals who work in optics. At the end we have also provided the school with an "Optics Education Directory", which is a comprehensive guide to optics courses and degree programs offered at educational institutions around the world ${ }^{13,14}$. This helps the students to choose the best school for their higher education in the field of optics and photonics.

\section{CONCLUSIONS}

In conclusion we have developed an advanced version of "El día de la luz" for high school students and have successfully implemented in various schools in the metropolitan area of Barcelona. This program focused on some important concepts of light illustrating ray and wave nature of light. Moreover, by means of simple experimental demonstrations, some very interesting applications show the conversion of optical energy from one form to the other. The simple demonstrations, not only explain the basic physics, but also its implementation in various applications used in our daily life. This event with active involvement of the students and teachers is very well appreciated. A video about "careers in optics" from SPIE and OSA is shown to motivate the students to pursue their further studies in optics. In addition an "Optics Education Directory" is provided to the teachers to help them in guiding the students, to choose interesting career path.

\section{ACKNOWLEDGEMENTS}

The authors acknowledge Prof. Lluis Torner, Director ICFO and chapter adviser, for his immense support in various aspects and his constant encouragement. We would like to acknowledge Prof. Jens Biegert and Prof. Valerio Pruneri, the advisers of ICFO-OSA and SPIE student chapter, respectively. We also acknowledge OSA and SPIE for their support in framing and implementing this event. "Optics Suitcase", the optics education kit from OSA Rochester section is gratefully acknowledged. Our thanks are also due to Dr. Silvia Carrasco, Laura Grau, and Tania Gómez for their administrative support. We would also like to acknowledge the ICONS members who volunteered to participate in the demonstrations: Goutam Samanta, Omid Kokabee, Florian Wolfgramm, Sukanya Randhawa, Daan Brinks, Saurabh Raj, Lars Neumann, Luis Martínez, Marina Mariano, Marta Castro, Roser Juanola, Jan Gieseler, Rafael Betancur, Alberto G. Curto and Sandro Perrone.

\section{REFERENCES}

[1] "Harnessing Light, Optical Science and Engineering in the 21st Century," National Academies of Science and Engineering,Washington,D.C., 1998. 
[2] Stephen M. Pompea and Tan Kah Gek, “Optics in the Great Exploration in Math and Science (GEMS) Program: A Summary of Effective Pedagogical Approaches” in Seventh International Conference on Education and Training in Optics and Photonics, Vol. 4588 (2002)

[3] Hannah D. Foreman, Francesca R. Parmigiani, Michal A. F. Roelens, and Robert E. Simpson, “The Lightwave Road show” in proceedings of The Education and Training in Optics and Photonics Conference (ETOP), ETOP035, 140 (2005)

[4] Osamu Takayama, Armand Niederberger, Petru Ghenuche, Manoj Mathew and Giovanni Volpe, "El día de la luz (the day of light)-two hours optics demonstration for secondary school students", in proceedings of The Education and Training in Optics and Photonics Conference (ETOP), ETOP, (2007)

[5] "Seeing the Light in Spain”, feature article in Optics \& Photonics News, Vol. 156 (2004)

[6] http://icons.icfo.es/

[7] http://ions-project.org/

[8] http://www.fascination-of-light.net/

[9] “PHOTONICS EDUCATION: Europe lights the way in photonics public outreach”, Laser Focus World, Vol. 45 (2009)

[10] Optics suitcase : http://www.opticsexcellence.org/Programs/EduOutreach/suitcasebroch.htm

[11] http://physics-animations.com/Physics/English/int_txt.htm

[12] http://spie.org/x30120.xml

[13] http://spie.org/x30118.xml

[14] http://www.opticseducation.org/ 